\title{
Visualising plural mappings of science for Sustainable Development Goals (SDGs)
}

\author{
Ismael Rafols ${ }^{1}$, Ed Noyons ${ }^{2}$, Hugo Confraria ${ }^{3}$ and Tommaso Ciarli ${ }^{4}$ \\ ${ }^{1}$ i.rafols@cwts.leidenuniv.nl \\ Centre for Science and Technology Studies (CWTS), Leiden University (The Netherlands) \& \\ Science Policy Research Unit (SPRU), University of Sussex, Brighton (England) \\ 2noyons@cwts.leidenuniv.nl \\ Centre for Science and Technology Studies (CWTS), Leiden University (The Netherlands) \\ ${ }^{3}$ h.confraria@sussex.ac.uk \\ Science Policy Research Unit (SPRU), University of Sussex, Brighton (England) \\ ${ }^{4}$ t.ciarli@sussex.ac.uk \\ Science Policy Research Unit (SPRU), University of Sussex, Brighton (England)
}

2021 Conference, International Society for Scientometrics and Informetrics (Accepted)

\begin{abstract}
Analysts are rapidly developing methods to map publications to SDGs in the face of policy demands. However, as reported by Armitage et al. (2020), a high degree of inconsistency is found when comparing the bibliometric corpora obtained with different approaches. These inconsistencies are not due to minor technical issues, but instead they represent different interpretations of SDGs. Given the variety of understandings regarding the relationship between research and SDGs, we propose that bibliometrics analysts should not assume that there is one single, preferred or consensus way of mapping SDGs to publications. We propose instead that, since different stakeholders have contrasting views about the relationships between science and SDGs, the contribution of bibliometrics should be to provide a plural landscape for stakeholders to explore their own views. We describe here the beta-version of an interactive platform that allows stakeholders to scrutinise in a global map of science the clusters potentially related to SDGs.
\end{abstract}

\section{Introduction}

The shift in S\&T policy from a focus on research quality towards societal impact has led to a demand for new S\&T indicators that capture the contributions of research to society (Wilsdon et al., 2017), in particular those aligned with SDGs. The use of the new 'impact' indicators would help monitoring if (and which) research organisations are aligning their research towards certain SDGs.

Responding to these demands data providers, consultancies and university analysts are rapidly developing methods to map projects or publications related to specific SDGs. These 'mappings' do not analyse the actual impact of research, but hope to capture instead if research is directed or related towards problems or technologies that can contribute to improving sustainability.

Yet this quick surge of news methods raises questions about the robustness of the mappings and indicators produced, and about the effects of using questionable indicators in policy making. The misuse of indicators in evaluation has been one of the key debates in science policy this last decade, as highlighted by initiatives such as the San Francisco Declaration on Research Assessment (2013) and the Leiden Manifesto (2015).

In a new study that aims to map publications to SDGs, we have found a high degree of inconsistency using different approaches, as recently reported by Armitage et al., (2020). In this contribution, we propose that these inconsistencies are not due to minor technical issues, but instead they represent different interpretations of SDGs. In other words, there is no a single 
objective 'truth' about which research is relevant for reaching SDGs. Since different stakeholders have contrasting and often conflicting views about the relationships between science and SDGs, the contribution of bibliometrics should be to provide a plural landscape for each stakeholder to explore which areas are 'really' related to SDGs according to his views (Rafols and Stirling, 2020). We describe here the beta-version of an interactive platform that allows stakeholders to explore in a global map of science the relationship between publications and SDGs.

\section{Recent efforts for mapping research to SDGs}

The first public analysis of SDG impact, released in 2020 by the Times Higher Education (THE, 2019), should be a motive for concern. For almost two decades, the THE has offered a controversial ranking of universities according to 'excellence'. The THE has now produced a new ranking of universities according to an indicator that sums dimensions of unclear relevance. For example, the indicator of the impact on health (SDG3) of a university depends on the one hand on its relative specialisation on health (as captured, e.g. by the proportion of papers related to health, $10 \%$ of total weight), and on the other hand on the proportion of health graduates (34.6\%). The score is also based on (self-reported) university policies such as care provided by the university, e.g. free sexual health services for students $(8.6 \%)$ or community access to sports (4\%). Such an heterogenous and arbitrary composite indicator is likely to cause more confusion than clarity and it is potentially harmful as it mystifies university policies for the SDGs.

One may thus believe that it is better to stick to objective measures such as 'relative specialisation on health' as captured by the proportion of papers related to health. In the THE ranking, this bibliometric data is supported by an Elsevier analysis of the publications that are related to the SDGs - which might seem more reliable than those based on data self-reported by universities (Jayabalasingham et al., 2019).

However, mapping publications to the SDGs is not straightforward. Bibliometricians are aware that while some consensus can be soon reached regarding the delineation of a traditional scientific fields, fields defined by policy issues such as environmental research are ambiguous. Following policy demands, there is currently a profusion of initiatives aiming at mapping publications to SDGs. The approaches developed by Bergen University (Armitage et al., 2020), Elsevier (Jayabalasingham, 2019), the Aurora Network, SIRIS Academic or the STRINGS project are based on searching for strings of keywords, in particular keywords found in the UN SDGs targets or other relevant policy documents. These searches are then enriched differently in each case. The hypothesis is that publications or projects containing these keywords are those best aligned with the UN SDG discourse. The question is then which keywords should be included, and which not. For example, why in some lists zika virus is included in the list of health SDG3, but not the closely related dengue virus, with a much higher disease burden?

An alternative approach being developed at NESTA and Dimensions (Wastl, 2020) uses policy documents and keywords to train machine learning algorithms in order to identify articles related to the SDGs instead of creating a list of keywords to search the articles. The alleged advantage of this algorithm is that machine learning is assumed to be more accurate than search strings. The downside of this approach is that is it a black box regarding the preferences (or biases) of the machine learning algorithms - and this is serious handicap for transparency.

In terms of consistency across approaches, an article recently published by a team at Bergen University sounded the alarm by showing that slightly different methods may produce extremely different results (Armitage et al., 2020). When comparing the papers related to SDGs retrieved with their own analysis with those by Elsevier, they found that there is astonishingly little overlap - in most SDGs only around 25\%-35\% as illustrated in Figure 1. The differences also affect the rankings of countries' contributions to the SDGs. The Bergen team concluded that "currently available SDG rankings and tools should be used with caution at their current 
stage of development.' We have conducted exploratory comparisons between the Bergen, SIRIS and STRINGS approach and can confirm major differences.
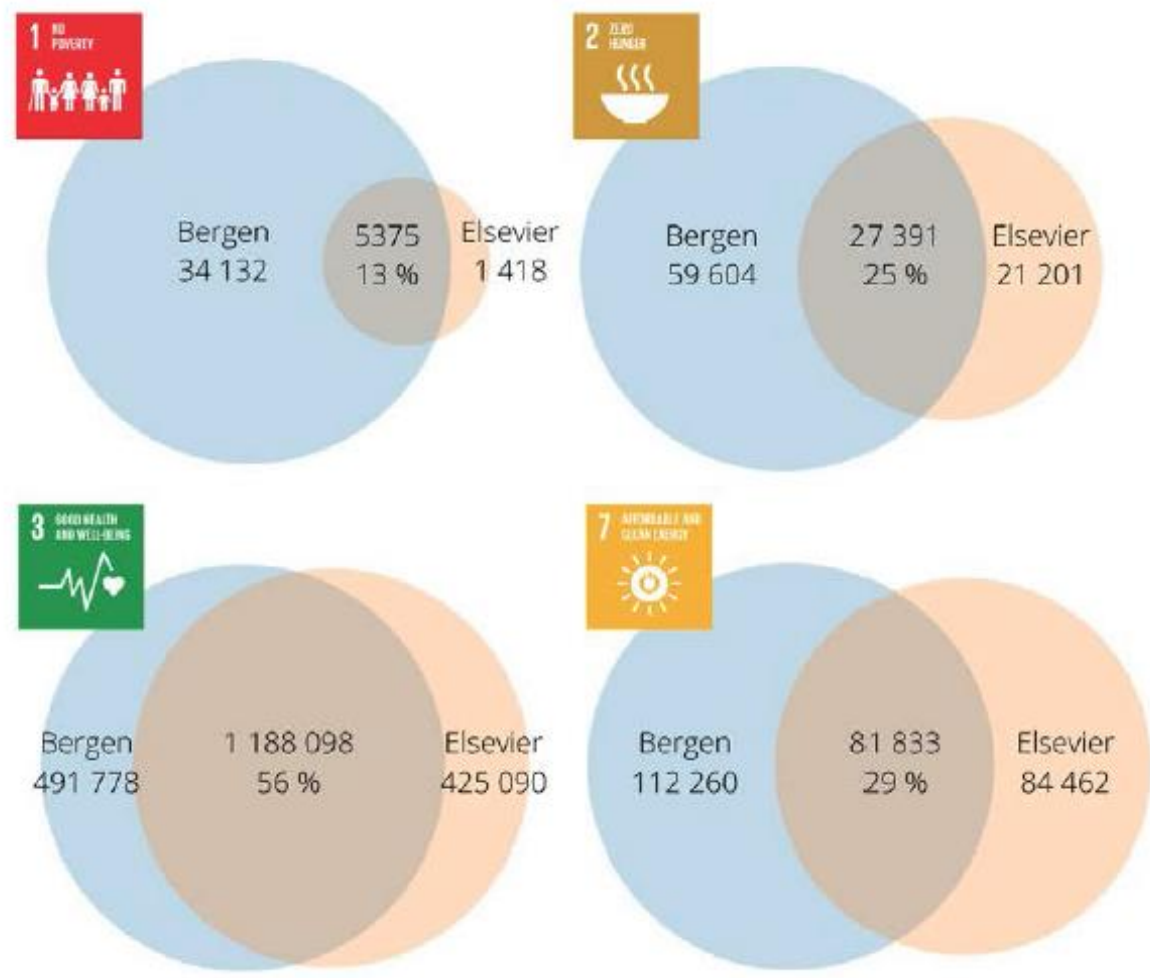

Figure 1. Comparison between Bergen and Elsevier approaches to mapping SDG-related publications. Based on WoS Core collection, 2015-2018. Source: Armitage et al. (2020).

\section{Conceptual framework: a multiplicity of possible mappings of SDGs}

However, from a conceptual point of view, perhaps we should not be surprised that different methods yield so different results. The SDGs refer to policy objectives in multiple dimensions - ending poverty, improving health, achieving gender equality, preserving the natural environment, etcetera. Innovation studies have shown that the social contributions of research are often unexpected and highly dependent on the local social contexts in which knowledges are created and used.

Nevertheless, most research is funded according to the expectations of the type of societal benefits that it may generate - and thus one can try to map these expectations or promises according to the language used in the (titles and abstracts of) projects and articles. However, the expected social contributions are often not made explicit in these technical documents because the experts reading them are assumed to know the potential applications.

As a consequence, the process of mapping projects or articles to the SDGs is ineluctably carried out through an interpretative process that 'translates' (or attempts to link) scientific discourse into potential societal outcomes. In other words, the analyst has to make assumptions regarding which terms are uniquely related to SDGs. Of course, such translation is dependent on the analysts' understandings of science and the SDGs. There is consensus on some of these understandings. For example, most analysts would agree that research on malaria is important for achieving global health. However, other translations are highly contested: should nuclear energy research be seen as a contribution to clean and affordable energy? Should all educational research be counted as contribution to the SDG on 'quality education'?

Furthermore, in a number of SDGs such as zero hunger (SDG 2) or reduced inequalities (SDG 10), there are stark disagreements on what the benefits of potential contributions: some stakeholders believe that GM crops (or organic farming) will help, while others believe that 
they will make the situation worse. Moreover, there is relatively little research explicitly mentioning issues such as gender or inequality in comparison to research whose innovation outcomes may affect these issues.

Another challenge is that many bibliometric databases used for analysis are not comprehensive, having a much larger coverage of traditional fields and rich countries (see Chapter 5 in Chavarro, 2017). This is particularly problematic, given that some research agendas relevant to developing countries are likely to be highly underrepresented (Rafols et al., 2015).

In summary, there is lack of consensus and many ambiguities on how publications relate to SDGs, and in these cases, the mappings will depend on the particular interpretation of the SDGs adopted in the methods for searching publications.

Given these ambiguities, we have developed an interactive mapping tool that aims to help stakeholders pick and choose which research lines they consider relevant in their context for each SDG. The map shows publication clusters of potentially relevant publication clusters for each SDGs with some interaction for users to understand the research contents of each cluster. This approach is introduced in the following sections.

\section{Data and methods}

We developed a search string extracting terms from policy documents explicitly mentioning SDGs. These search-strings were applied to retrieve publications (articles and reviews) for the period 2015-2019 from the CWTS in-house version of Web of Science (WoS) after an iterative process of refinement to avoid false negatives. This process was informed by comparisons with searches carried out by other initiatives, in particular SIRIS and Bergen. Details of this retrieval methodology are presented in the 2021 ISSI proceeding by Confraria et al.

On the other hand, we rely on an article-level classification based on direct-citation clustering of about 4,000 categories. These clusters are positioned in a science map based on citations across clusters according to a VOSviewer layout. This information is imported on a Tableau visualisation interface. The Tableau interface shows the clusters retrieved for each SDG with information on the cluster and the publications related to SDGs, as detailed below.

\section{Results: a visualisation interface to explore plural mappings of SDGs}

In this proceeding we introduce the beta-version of a visualisation interface aimed at facilitating the exploration of publication clusters related to SDG. The interface allows to choose one SDG (top right). Then the user can set the thresholds for considering if a cluster is related to this particular SDG: the minimum number of publications retrieved by the search string of this SDG (seed publications), and the minimum share of seeds in a cluster.

The clusters with figures above these thresholds are displayed in a science map according to their position in the global map of science. The colours of the clusters represent broad disciplines. Clusters in Social science and humanities (blue) are in the top left, Biomedicine and health (yellow) are in the left, Physical sciences and engineering (red) are in the bottom right, Maths and computer sciences (green) are in the right, and Life and earth sciences (light-blue) are in the centre-left.

The contents of specific clusters can be explored by placing the pointer of the mouse over the cluster of interest. For each cluster, you can see the 5 most relevant labels (where the labels are terms with high frequency and high cluster specificity) and the 5 most frequent journals. By clicking on the node or by clicking on the corresponding row in the list of 'Selected communities', the right column 'Community Pubs' allows to click into specific publications, which are linked via DOI to website of the publication. In this way, it is possible to read titles and abstracts and better understand the content of clusters.

Let us take for example, the cluster labelled as 'greater sage grouse' (centre-left) in SDG 7 (energy). This may seem a false positive since a grouse is a type of bird, rather than energy. 
However, the other labels of the cluster are wind farm and turbine. By clicking at the community publications, we then see that the article contents is related to bird collisions with wind turbines and power lines. Interestingly this cluster is about energy, but it shows a trade-off between SDG7 (Energy) and SDG15 (Life on Land).

SDG 7 - Ensure access to affordable, reliable, sustainable and modern energy for all
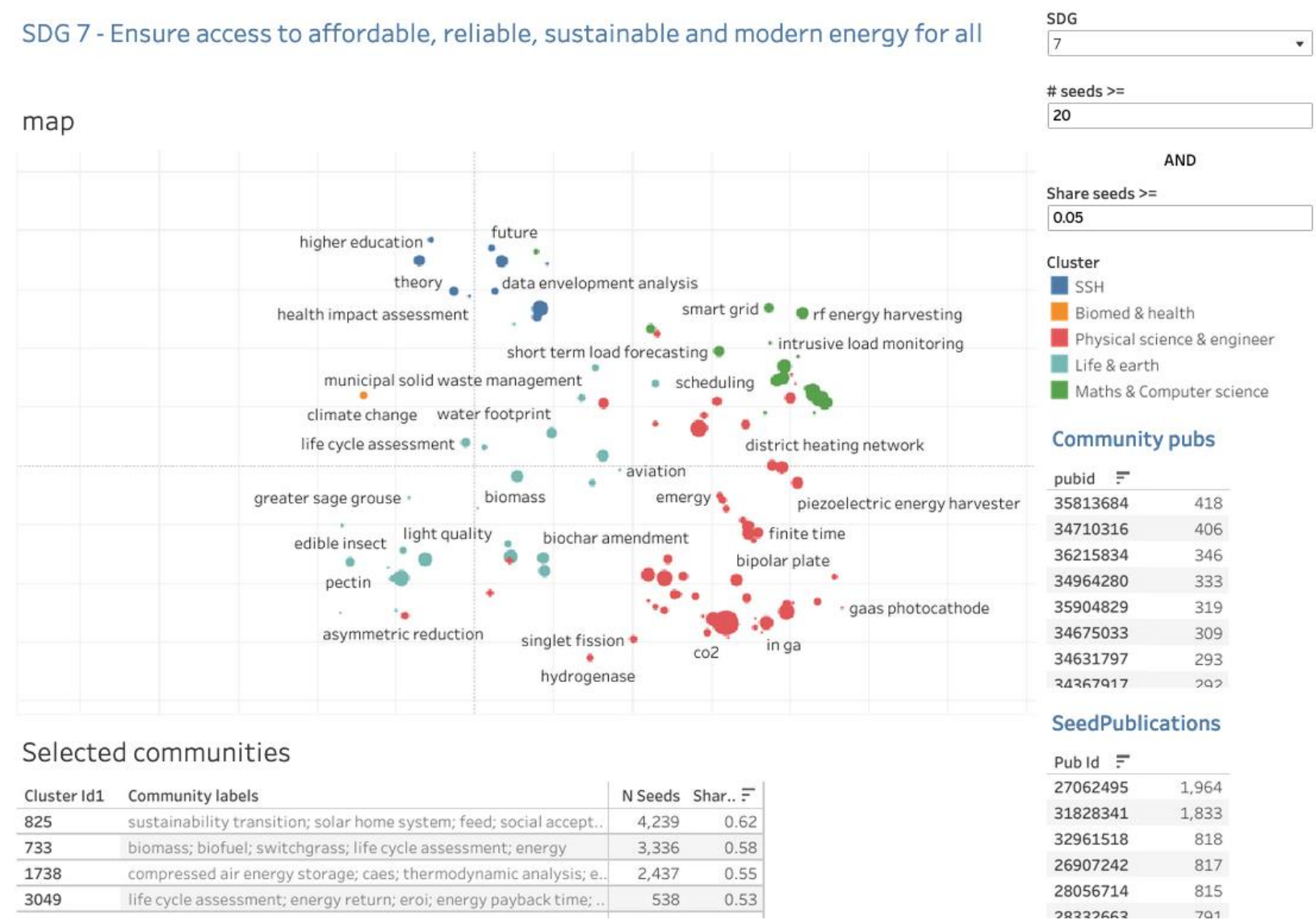

\section{Selected communities}

\begin{tabular}{ll|rr} 
Cluster Id1 & Community labels & N Seeds & Shar.. $\bar{~}$ \\
\hline $\mathbf{8 2 5}$ & sustainability transition; solar home system; feed; social accept.. & 4,239 & 0.62 \\
\hline 733 & biomass; biofuel; switchgrass; life cycle assessment; energy & 3,336 & 0.58 \\
\hline $\mathbf{1 7 3 8}$ & compressed air energy storage; caes; thermodynamic analysis; e.. & 2,437 & 0.55 \\
\hline $\mathbf{3 0 4 9}$ & lifecycle assessment; energy return; eroi; energy payback time;... & 538 & 0.53 \\
\hline
\end{tabular}

Figure 1. Interactive visualisation interface of the STRINGS project for SDG7 (Energy). It shows clusters with at least 20 and $5 \%$ of its publications having terms related to SDG7. Cluster labels are shown at the bottom left. Instances of publications of a cluster and those retrieve (seeds) shown in the right columns. Available at: https:/public.tableau.com/profile/ed.noyons\#!/vizhome/UKStringsSDGtocommunities/Dashboard1

The map illustrates the many different types of research trajectories that may be related to sustainable energy, from higher education (top left), to smart grid management (top right) to thin solid films for photovoltaics made out of copper indium gallium selenide solar cells (InGa, bottom right), to diesel engines (centre-right) and alternative fuels in aviation (centre). This large variety of research trajectories shows that science can contribute to sustainable energy in very different (sometimes conflicting) innovation pathways.

However, stakeholders in specific contexts may think that some research trajectories are not as relevant for them as other trajectories. For example, developing countries might object to considering that cancer research as a priority for SDGs, given the relative under-investment in infectious diseases - the latter issues having a much higher burden for them. Therefore, the proposal of an interactive map is aimed at facilitating that different stakeholders find and choose those research trajectories that they consider y relevant for SDGs. Instead of having one single delineation of each SDG, the use of the interactive visualisation by stakeholders makes it possible to develop a plural and conditional mapping between SDGs and publications (Rafols and Stirling, 2020). 
We can thus think of research over a given SDG in terms of a variable portfolio of research options as shown by clusters, with different stakeholders having different perspectives, values and needs regarding the balance between options, and the options that should be prioritized (Wallace and Rafols, 2015; Ciarli and Rafols, 2019). A given SDG will not be achieved by having more research on this SDG - but by having a balance of research options that is conducive to achieving the SDGs. In contrast, we think that having only rankings of universities according to their SDG is not a meaningful source of information for policy decisions.

\section{Conclusion}

In summary, given the variety of understandings regarding the relationship between research and SDGs, we propose that bibliometrics analysts should not assume that there is one single, preferred or consensus way of mapping SDGs to publications. Such assumption is likely to reproduce hegemonic perspectives on SDGs - often given by rich countries at influential institutional actors (Stirling, 2019). Instead, we propose a mapping tool which suggests publication clusters potentially related to a given SDG, thus allowing plural interpretations.

\section{Acknowledgments}

This work is part of the STRINGS (STeering Research INnovation for Global GoalS). We are indebted to other members of the project for fruitful discussions.

\section{References}

Armitage, C. S., Lorenz, M., \& Mikki, S. (2020). Mapping scholarly publications related to the Sustainable Development Goals: Do independent bibliometric approaches get the same results? Quantitative Science Studies, 1(3), 1092-1108.

Chavarro, Diego (2017) Universalism and particularism: explaining the emergence and growth of regional journal indexing systems. Doctoral thesis $(\mathrm{PhD})$, University of Sussex. Retrieved on July 11 July 2020 from: http://sro.sussex.ac.uk/id/eprint/66409/

Ciarli, T., \& Ràfols, I. (2019). The relation between research priorities and societal demands: The case of rice. Research Policy, 48(4), 949-967.

DORA (2013) San Francisco Declaration on Research Assessment. Retrieved January 24, 2021 from: https://sfdora.org/

Jayabalasingham, B., Boverhof, R., Agnew, K., Klein, L. (2019) Identifying research supporting the United Nations Sustainable Development Goals. Elsevier documentation. DOI: $\underline{10.17632 / 87 \mathrm{txkw} 7 \mathrm{khs} .1}$

Rafols, I., Ciarli, T., \& Chavarro, D. (2015) Under-reporting research relevant to local needs in the global south. Database biases in the representation of knowledge on rice. Retrieved February 9, 2021 from: https://osf.io/preprints/socarxiv/3kf9d/

Rafols, I., \& Stirling, A. (2020). Designing indicators for opening up evaluation. Insights from research assessment. Retrieved February 9, 2021 from: https://osf.io/preprints/socarxiv/h2fxp/

Stirling, A. (2019). How deep is incumbency? A 'configuring fields' approach to redistributing and reorienting power in socio-material change. Energy Research \& Social Science, 58, 101239.

THE (2020) Impact rankings. Retrieved February 25, 2021 from: https://www.timeshighereducation.com/impactrankings

Wallace, M. L., \& Rafols, I. (2015). Research portfolio analysis in science policy: moving from financial returns to societal benefits. Minerva, 53(2), 89-115.

Wastl, Juergen; Porter, Simon; Draux, Hélène; Fane, Briony; Hook, Daniel (2020): Contextualizing Sustainable Development Research. Digital Science. Report. https://doi.org/10.6084/m9.figshare.12200081.v2

Wilsdon, J. R., Bar-Ilan, J., Frodeman, R., Lex, E., Peters, I., \& Wouters, P. (2017). Next-generation metrics: Responsible metrics and evaluation for open science. European Commission, Brussels. https://ec.europa.eu/research/openscience/pdf/report.pdf 